\title{
Bioinformatic analysis and characteristics of glycoprotein $C$ encoded by the newly identified $U L 44$ gene of duck plague virus
}

\author{
K.F. Sun ${ }^{1,2,3}$, A.C. Cheng ${ }^{1,2,3}$ and M.S. Wang ${ }^{1,2,3}$ \\ ${ }^{1}$ Institute of Preventive Veterinary Medicine, Sichuan Agricultural University, \\ Chengdu City, Sichuan, China \\ ${ }^{2}$ Avian Disease Research Center, \\ College of Veterinary Medicine of Sichuan Agricultural University, \\ Ya'an, Sichuan, China \\ ${ }^{3}$ Key Laboratory of Animal Disease and Human Health of Sichuan Province, \\ Sichuan Agricultural University, Chengdu City, Sichuan, China \\ Corresponding authors: A.C. Cheng / M.S. Wang \\ E-mail: chenganchun@vip.163.com / mshwang@163.com
}

Genet. Mol. Res. 13 (2): 4505-4515 (2014)

Received January 30, 2013

Accepted June 17, 2013

Published June 17, 2014

DOI http://dx.doi.org/10.4238/2014.June.17.2

\begin{abstract}
Glycoprotein C is one of the duck plague virus (DPV) glycoproteins and is encoded by the DPV UL44 gene. DPV glycoprotein $\mathrm{C}$ (DPV-gC) comprises 431 amino acids with a putative molecular mass of $47.35 \mathrm{kDa}$. Sequence analysis indicated that the protein possesses typical characteristics of type-I membrane glycoproteins, containing an $\mathrm{N}$-terminal signal sequence, an external domain, a C-terminal membrane anchor region, and a short cytoplasmic domain. Comparisons of 22 alphaherpesvirus-gC protein sequences revealed eight conservative Cys-residue sites, which may play a crucial role in the biological functions and structural stabilization of the DPV-gC protein. Estimates of potential antigenic epitopes and secondary structure identified four B cell dominant epitopes, which are located at amino acids 68-71, 8791, 369-352, and 372-374. A model for the structure of DPV-gC was derived by associating its predicted secondary and three-dimensional
\end{abstract}


structures. In conclusion, these results will provide a basis for further functional studies of DPV-gC, establishing novel clinical diagnoses of $\mathrm{DPV}$, and in the development of a new DPV vaccine.

Key words: Bioinformatics; Structure; Function; Glycoprotein C; Duck plague virus

\section{INTRODUCTION}

Duck plague virus (DPV), which is the causative agent of duck plague has been classified in the subfamily Alphaherpesvirinae of Herpesviridae based on the report of the ninth International Committee on Taxonomy of Viruses (ICTV), but has not yet been grouped into any genus (Davison et al., 2009; King et al., 2011). DPV has a typical herpes virion, consisting of a linear double-stranded DNA core, an icosahedral capsid, a tegument, and an envelope containing viral glycoprotein spikes on its surface (Pellett and Roizman, 2007; Sandhu and Shawky, 2008). In duck breeding areas of the world where the disease has been reported, DPV has caused significant economic losses in both domestic and wild waterfowl due to high mortality and low egg production rates (Sandhu and Shawky, 2008).

As a multifunctional glycoprotein in Alphaherpesvirinae, glycoprotein $\mathrm{C}$ is involved in viral attachment, release, virulence, and immune evasion, among other functions (Schreurs et al., 1988; Friedman et al., 1996; Chowdhury et al., 2000; Jarosinski and Osterrieder, 2010). Being situated on the envelope surface of mature virus particles, glycoprotein $\mathrm{C}$ contains many antigen determinants, and can adequately induce the immune response (Ben-Porat et al., 1986; Guo et al., 1989; Tewari et al., 1995). In the DPV genome, the UL44 gene, which is conserved with the UL44 gene of other alphaherpesviruses (Allen and Coogle, 1988; Fitzpatrick et al., 1989; Nicolson and Onions, 1990), was identified and sequenced in our laboratory. The complete nucleotide sequence of the UL44 gene from the DPV CHv strain has been submitted in the GenBank database and was assigned the accession number EU076811. We named the corresponding gene as DPV-UL44 and the protein as DPV-gC.

The objective of the present study was to provide information about the structure and functions of DPV-gC. This study may provide insight for further research related to DPV-gC, and provide a fundamental basis for establishing new types of clinical diagnoses of DPV and for the development of a new DPV vaccine.

\section{MATERIAL AND METHODS}

\section{Prediction of the physicochemical properties of DPV-gC}

The DNAStar 7.1 program was used to analyze components of the DPV-gC sequence. The deduced amino acid sequence was analyzed with online prediction tools. The signal peptide, phosphorylation site, transmembrane region, motifs, hydrophobicity, subcellular localization, and protein-protein interaction sites were analyzed with the programs SignalP 4.0 (http://www.cbs.dtu.dk/services/SignalP; Petersen et al., 2011), NetPhos 2.0 (http://www.cbs. dtu.dk/services/NetPhos/; Blom et al., 1999), TMHMM (http:/genome.cbs.dtu.dk/services/ TMHMM/), PSORT (http://www.psort.org/psortb/results.pl; Yu et al., 2010), and PredictPro- 
tein (http://www.predict-protein.org/submit.php), respectively.

\section{Estimation of potential antigenic epitopes}

To estimate the potential antigenic epitopes of DPV-gC, the amino acid sequence of the DPV-gC was analyzed using online prediction tools and commensurate software. Antigenic peptide prediction, hydrophilicity estimation, surface accessibility, and flexibility assumption were performed using prediction tools. An online web server (http://immunax.dfci.harvard. edu/Tools/antigenic.pl) provided a pathway to predict sequences of peptides within a protein that were likely to be antigenic by eliciting an antibody response. The SIB Bioinformatics Resource Portal (http://us.expasy.org/tools/protscale.html) was used to predict the hydrophilicity of DPV-gC, which was based on the method of Kyte \& Doolittle (Kyte and Doolittle, 1982). The PredictProtein program (http://www.predictprotein.org/submit.php) was used to predict the surface accessibility of DPV-gC. The flexibility was DPV-gC was determined with the Protean procedure in DNAStar 7.1, based on methods of Karplus-Schulz (Karplus and Schulz, 1985).

\section{Comparison of the DPV-gC sequence with 21 published gC protein sequences and phylogenetic tree construction}

We obtained information of the 21 published $\mathrm{gC}$ amino acid sequences of other alphaherpesviruses from the National Center for Biotechnology Information (NCBI) database (Table 1). Comparison and analysis of the conserved regions of DPV-gC with the 21 published alphaherpesvirus-gC amino acid sequences was performed using ClustalX 1.83 or DNAStar 7.1. The phylogenetic tree was constructed using the neighbor-joining method in PHYLIP 3.69. The reliability of each tree branch was tested by bootstrap analysis of 100 data samples and the trees were constructed using TreeView 1.66.

\begin{tabular}{|c|c|c|c|c|c|}
\hline Genus & Virus name (abbreviation) & $\begin{array}{l}\text { GenBank } \\
\text { Accession No. }\end{array}$ & $\begin{array}{l}\text { CDS Sequence } \\
\text { length (bp) }\end{array}$ & Protein ID & $\begin{array}{l}\text { Protein } \\
\text { length (aa) }\end{array}$ \\
\hline \multirow[t]{10}{*}{ Varicellovirus } & Bovine herpesvirus 1 (BoHV-1) (IBRV) & NC_001847.1 & 1527 & NP_045314.1 & 508 \\
\hline & Bovine herpesvirus 5 (BoHV-5) & $\mathrm{NC}^{-} 005261.2$ & 1461 & NP 954903.1 & 486 \\
\hline & Cercopithecine herpesvirus 9 (CeHV-9) (SVV) & $\mathrm{NC}-002686.2$ & 1623 & NP 077429.1 & 540 \\
\hline & Equid herpesvirus 1 (EHV-1) (EAV) & $\mathrm{NC} 001491.2$ & 1407 & YP_053061.1 & 468 \\
\hline & Equid herpesvirus 4 (EHV-4) & $\mathrm{NC}^{-} 001844.1$ & 1458 & $\mathrm{NP}^{-} 045233.1$ & 485 \\
\hline & Equid herpesvirus 8 (EHV-8) & NC 017826.1 & 1431 & YP 006272995.1 & 476 \\
\hline & Equid herpesvirus 9 (EHV-9) & NC_011644.1 & 1407 & YP_002333497.1 & 468 \\
\hline & Felid herpesvirus 1 (FHV-1) & NC_- 013590.2 & 1605 & YP_003331535.1 & 534 \\
\hline & Human herpesvirus 3 (HHV-3) (VZV) & $\mathrm{NC} 001348.1$ & 1683 & NP 040137.1 & 560 \\
\hline & Suid herpesvirus 1 (SuHV-1) (PRV) & $\mathrm{NC} 006151.1$ & 1440 & YP 068347.1 & 458 \\
\hline \multirow{6}{*}{ Simplexvirus } & Cercopithecine herpesvirus 1 (CeHV-1) (B virus) & $\mathrm{NC}^{-} 004812.1$ & 1404 & NP 851904.1 & 467 \\
\hline & Cercopithecine herpesvirus 2 (CeHV-2) (SA8) & $\mathrm{NC}^{-} 006560.1$ & 1395 & YP 164487.1 & 464 \\
\hline & Cercopithecine herpesvirus 16 (CeHV-16) (HVP-2) & $\mathrm{NC}-007653.1$ & 1389 & YP 443891.1 & 462 \\
\hline & Human herpesvirus 1 (HHV-1) (HSV-1) & NC_001806.1 & 1536 & NP_044646.1 & 511 \\
\hline & Human herpesvirus 2 (HHV-2) (HSV-2) & $\mathrm{NC}^{-} 001798.1$ & 1443 & NP 044514.1 & 480 \\
\hline & Saimiriine herpesvirus 1 (SaHV-1) & $\mathrm{NC}^{-} 014567.1$ & 1614 & YP 003933796.1 & 537 \\
\hline \multirow{2}{*}{ Iltovirus } & Gallid herpesvirus 1 (GaHV-1) (ILTV) & $\mathrm{NC}^{-} 006623.1$ & 1245 & YP 182373.1 & 414 \\
\hline & Psittacid herpesvirus 1 (PsHV-1) & NC_005264.1 & 1389 & NP 944417.1 & 462 \\
\hline \multirow{3}{*}{ Mardivirus } & Gallid herpesvirus 2 (GaHV-2) (MDV-1) & $\mathrm{NC}^{-} 002229.3$ & 1506 & YP 001033973.1 & 501 \\
\hline & Gallid herpesvirus 3 (GaHV-3) (MDV-2) & $\mathrm{NC}^{-} 002577.1$ & 1437 & NP 066876.1 & 478 \\
\hline & Meleagrid herpesvirus 1 (MeHV-1) (HVT) & NC_002641.1 & 1470 & NP_073338.1 & 489 \\
\hline
\end{tabular}




\section{Secondary and tertiary structure prediction}

The presumed alpha helix, extended strand, and random coil of DPV-gC were obtained from the Network Protein Sequence Alignment (NPSA) (http://npsa-pbil.ibcp.fr/cgibin/secpred_mlr.pl; Combet, et al., 2000). The prediction of the 3D structure of the DPV-gC protein was performed through the SWISS-MODEL server (http://www.cbs.dtu.dk/services/ CPHmodels/; Kelley and Sternberg, 2009).

\section{RESULTS}

\section{Physico-chemical properties of DPV-gC}

The DPV UL44 gene was originally obtained by sequencing the DPV genomic library that was constructed in our laboratory. The predicted open reading frame of the DPV UL44 gene was expected to encode a protein comprising 431 amino acids with a putative molecular mass of $47.35 \mathrm{kDa}$ and an isoelectric point (pI) of 5.29. DNAStar7.1 analysis revealed that the polypeptide encoded by the DPV UL44 gene was composed of $33.64 \%$ hydrophobic amino acids, $29.470 \%$ hydrophilic amino acids, $8.12 \%$ strongly basic amino acids, and $10.44 \%$ strongly acidic amino acids.

Furthermore, using different online web servers, we obtained more detailed information about the structure and function of DPV-gC. First, the N-terminus of the putative protein containing four potential N-linked glycosylation sites (at aa residues 52, 58, 163, and 279) and three $\mathrm{N}$-myristoylation sites (at aa residues 51, 90, and 406) could be clearly identified. Second, one cAMP- and cGMP-dependent protein kinase phosphorylation site (at aa residue 166), nine protein kinase $C$ phosphorylation sites (at aa residues 32, 162, 165, 176, 213, 230, 236, 264, and 365), and five casein kinase II phosphorylation site (at aa residues 77, 105, 142, 239, and 254) were identified in DPV-gC. Furthermore, amino acid sequence analysis revealed that DPV-gC is a type-I membrane glycoprotein that can be divided into three kinds of distinct functional domains: the extracellular domain, the hydrophobic transmembrane domain, and the cytoplasmic domain.

\section{Estimated potential antigenic epitopes}

The prediction tools of online web servers indicated that the DPV-gC amino acid sequence contains 20 main antigenic determinants, which were situated in amino acids 26-39, 5978, 85-91, 94-101, 132-141, 148-158, 171-197, 199-207, 216-222, 227-233, 242-254, 262-267, 279-296, 298-316, 332-343, 351-357, 359-370, 372-386, 395-406, and 408-423. Hydrophilicity prediction revealed that the highest points of DPV-gC's hydrophilicity were between amino acids 20-28, 29-59, 66-71, 80-97, 104-133, 143-149, 157-171, 194-203, 209-215, 217-219, 221-226, 231-243, 257-263, 268-278, 300-302, 321-331, 341-344, 346-377, 382-393, and 421427. Due to their exposure on the virion or cell membrane surface, these parts of the protein are likely to contain the major antigenic epitopes of DPV-gC. Results obtained from the surface accessibility website indicated that the DPV-gC amino acid sequence contains 21 main surface accessibility domains, which are situated in amino acids 29-34, 50-60, 68-82, 86-93, 104-124, 127-133, 142-146, 155-166, 170-174, 181-185, 206-213, 220-232, 234-243, 256-262, 270-280, 
309-313, 320-333, 342-346, 369-377, 387-393, and 423-431. In addition, the DNAStar 7.1 software revealed that DPV-gC contained 24 main flexibility domains, which located at amino acids 19-24, 31-35, 40-43, 48-57, 68-71, 87-98, 104-112, 119-122, 127-133, 140-147, 154-170, 181-186, 196-199, 210-215, 221-226, 230-243, 252-263, 270-277, 322-327, 344-352, 358-362, 370-374, 385-394, and 424-428. Associating four parameters (hydrophilicity, surface properties, flexibility, and main antigenic determinants) identified potential antigenic epitopes, which were situated at amino acids 31-34, 68-71, 87-91, 369-352, and 372-374.

\section{Comparing the 22 alphaherpesvirus-gC protein sequences and constructing the phylogenetic tree}

Comparison of the 22 alphaherpesvirus-gC protein sequences by DNAStar7.1 indicated that there were eight conserved Cys-residue sites. Combined with the results of signal peptide and transmembrane domain prediction of the $\mathrm{gC}$ protein, statistical results of the interval among the conserved Cys-residue sites are shown in Table 2. Except for ILTV and PsHV-1 from the genus Iltovirus, the biggest difference in sequence length was observed in the interval between the signal peptide and the conserved Cys-residue site 1, followed by the length of the cytoplasmic domain and the interval between the conserved Cys-residue sites 2 and 3 .

\begin{tabular}{|c|c|c|c|c|c|c|c|c|c|c|c|c|}
\hline Virus name (abbreviation) & $\begin{array}{r}\text { Signal } \\
\text { peptide }\end{array}$ & & $\mathrm{C} 1-\mathrm{C} 2$ & $\mathrm{C} 2-\mathrm{C} 3$ & $\mathrm{C} 3-\mathrm{C} 4$ & $\mathrm{C} 4-\mathrm{C} 5$ & $\mathrm{C} 5-\mathrm{C} 6$ & C6-C7 & $\mathrm{C} 7-\mathrm{C} 8$ & & $\begin{array}{l}\text { Transmembrane } \\
\text { regions }\end{array}$ & $\begin{array}{l}\text { Intracellular } \\
\text { region }\end{array}$ \\
\hline $\begin{array}{l}\text { Bovine herpesvirus } 1 \\
\text { (BoHV-1) (IBRV) }\end{array}$ & 26 & 115 & 16 & 130 & 60 & 38 & 3 & 26 & 25 & 27 & 23 & 11 \\
\hline Bovine herpesvirus 5 (BoHV-5) & 26 & 94 & 16 & 130 & 60 & 38 & 3 & 25 & 25 & 28 & 23 & 10 \\
\hline $\begin{array}{l}\text { Cercopithecine herpesvirus } 9 \\
\text { (CeHV-9) (SVV) }\end{array}$ & 20 & 124 & 16 & 160 & 60 & 38 & 3 & 27 & 25 & 29 & 23 & 6 \\
\hline Equid herpesvirus 1 (EHV-1) (EAV) & 27 & 48 & 16 & 145 & 61 & 38 & 3 & 28 & 25 & 32 & 23 & 14 \\
\hline Equid herpesvirus 4 (EHV-4) & 27 & 56 & 16 & 146 & 61 & 38 & 3 & 28 & 25 & 32 & 23 & 14 \\
\hline Equid herpesvirus 8 (EHV-8) & 27 & 56 & 16 & 145 & 61 & 38 & 3 & 28 & 25 & 32 & 23 & 13 \\
\hline Equid herpesvirus 9 (EHV-9) & 27 & 48 & 16 & 145 & 61 & 38 & 3 & 28 & 25 & 32 & 23 & 14 \\
\hline Felid herpesvirus 1 (FHV-1) & 27 & 117 & 17 & 147 & 60 & 38 & 3 & 30 & 25 & 33 & 23 & 6 \\
\hline $\begin{array}{l}\text { Human herpesvirus } 3 \\
\text { (HHV-3) (VZV) }\end{array}$ & 21 & 136 & 16 & 166 & 61 & 38 & 3 & 27 & 25 & 29 & 23 & 6 \\
\hline Suid herpesvirus 1 (SuHV-1) (PRV) & 20 & 82 & 16 & 135 & 69 & 38 & 3 & 22 & 25 & 33 & 23 & 5 \\
\hline $\begin{array}{l}\text { Cercopithecine herpesvirus } 1 \\
\text { (CeHV-1) (B virus) }\end{array}$ & 31 & 44 & 16 & 137 & 60 & 38 & 3 & 27 & 23 & 35 & 23 & 22 \\
\hline $\begin{array}{l}\text { Cercopithecine herpesvirus } 2 \\
\text { (CeHV-2) (SA8) }\end{array}$ & 25 & 43 & 16 & 141 & 60 & 38 & 3 & 27 & 23 & 35 & 23 & 22 \\
\hline $\begin{array}{l}\text { Cercopithecine herpesvirus } 16 \\
\text { (CeHV-16) (HVP-2) }\end{array}$ & 22 & 43 & 16 & 142 & 60 & 38 & 3 & 27 & 23 & 35 & 23 & 22 \\
\hline Human herpesvirus 1 (HHV-1) (HSV-1) & 24 & 102 & 16 & 141 & 60 & 38 & 3 & 28 & 22 & 35 & 23 & 11 \\
\hline Human herpesvirus 2 (HHV-2) (HSV-2) & 27 & 102 & 16 & 141 & 60 & 38 & 3 & 27 & 23 & 35 & 23 & 11 \\
\hline Saimiriine herpesvirus 1 (SaHV-1) & 19 & 140 & 16 & 138 & 63 & 38 & 3 & 27 & 23 & 32 & 23 & 7 \\
\hline Gallid herpesvirus 1 (GaHV-1) (ILTV) & 22 & 4 & 16 & 248 & 60 & 0 & 0 & 0 & 0 & 30 & 23 & 5 \\
\hline Psittacid herpesvirus 1 (PsHV-1) & 23 & 43 & 17 & 257 & 0 & 0 & 6 & 31 & 21 & 31 & 23 & 3 \\
\hline Gallid herpesvirus 2 (GaHV-2) (MDV-1) & 1) 27 & 82 & 16 & 127 & 61 & 36 & 3 & 28 & 25 & 32 & 23 & 9 \\
\hline Gallid herpesvirus 3 (GaHV-3) (MDV-2) & 2) 27 & 56 & 16 & 127 & 61 & 37 & 3 & 28 & 25 & 29 & 23 & 15 \\
\hline $\begin{array}{l}\text { Meleagrid herpesvirus } 1 \\
\text { (MeHV-1) (HVT) }\end{array}$ & 31 & 70 & 16 & 150 & 61 & 37 & 3 & 28 & 25 & 33 & 23 & 4 \\
\hline Anatid herpesvirus 1 (AnHV-1) (DPV) & 22 & 7 & 16 & 155 & 61 & 37 & 3 & 32 & 25 & 32 & 23 & 10 \\
\hline
\end{tabular}

The amino acid sequence alignment based on truncated $\mathrm{gC}$ protein sequences (for which the residues before the conserved Cys-residue site 1 were deleted) showed that the Cys- 
residue sites 1, 2, 5, and 8, along with three Pro-residue, three Trp-residue, two Tyr-residue, and one Gly-residue were all completely conserved in alphaherpesviruses. In particular, there was a conservative module $\left(\mathrm{Y}^{*} \mathrm{C}^{* * *} \mathrm{G}\right)$ around the conserved Cys-residue site 8 in alphaherpesviruses. Moreover, there were also conservative modules found around Cys-residue conservative sites 3, 4, 5, 6, and 7, except for ILTV and PsHV-1 from the genus Iltovirus.

The phylogenetic tree was constructed by comparing the truncated DPV-gC amino acid sequence with 21 truncated $\mathrm{gC}$ amino acid reference sequences of the other alphaherpesviruses. As shown in Figure 1, the $22 \mathrm{gC}$ amino acid sequences were divided into four branches, which were respectively termed Mardivirus (which contains HVT, MDV-1, and MDV-2), Iltovirus (which contains ILTV and PsHV-1), Simplex virus (which contains CeHV1, CeHV-2, HVP-2, HSV-1, HSV-2, and SaHV-1), and Varicello virus (which contains BoHV1, BoHV-5, CeHV-9, EHV-1, EHV-4, EHV-8, EHV-9, FHV-1, PRV, and VZV). Further observation of the phylogenetic branching showed that DPV-gC was closely related to sequences in the Mardivirus genus. Notably, however, the DPV sequence clustered within a monophyletic clade, which was independent of the others.

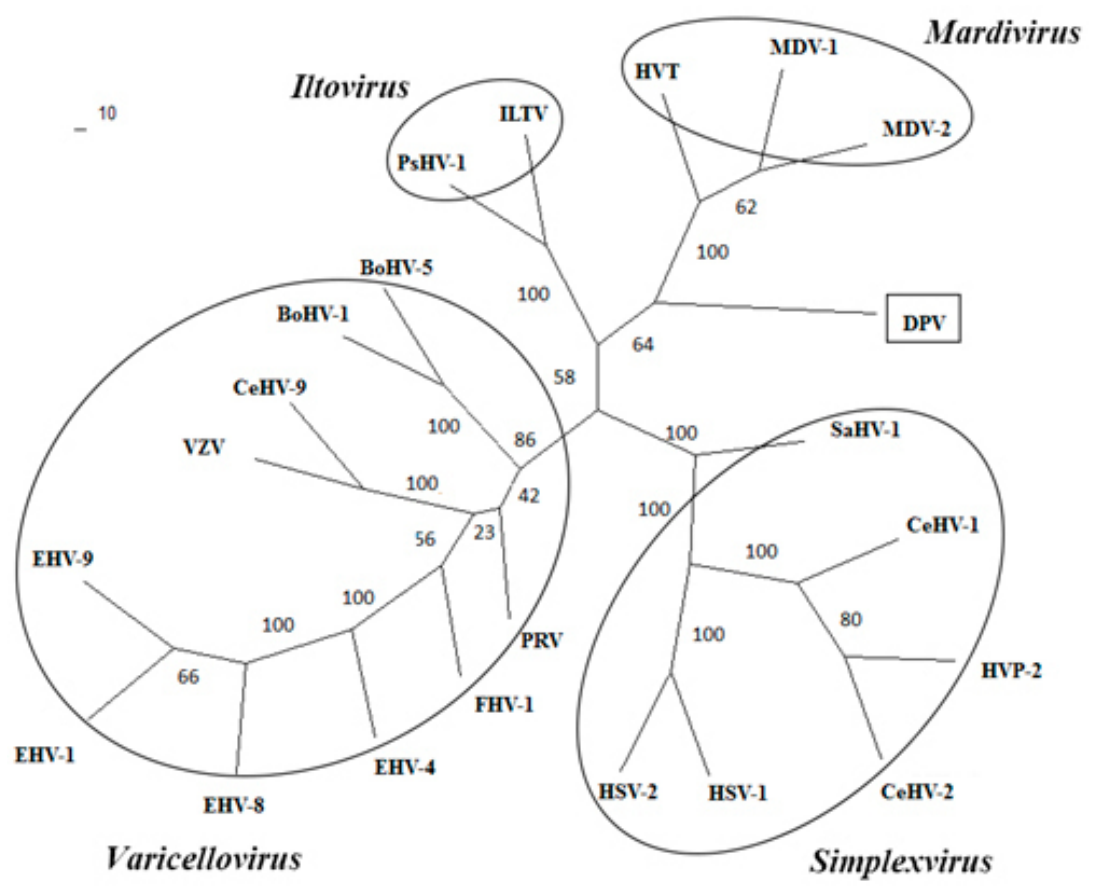

Figure 1. Phylogenetic analysis of the truncated DPV-gC amino acid sequence and 21 truncated gC amino acid referenced sequences of the other alphaherpesviruses was aligned using ClustalX 1.83. Phylogenetic tree was constructed by using Phylip 3.69 and drawn using TreeView 1.66.

\section{Secondary and tertiary structure prediction}

The prediction of DPV-gC's secondary structure is shown in Figure 2. The result indicated that $9.51,32.95$, and $57.54 \%$ of the DPV-gC protein consists of an alpha helix (H), an 
extended strand (E), and a random coil (C), respectively. The prediction of the 3D structure of the DPV-gC protein was determined with the SWISS-MODEL database (Figure 3). Three hundred sixty-three residues ( $84 \%$ of DPV-gC) were modeled with $99.8 \%$ confidence by the single highest scoring template. These 363 residues clearly represented the extracellular domain truncated signal peptide of DPV-gC. By associating the predicted secondary and 3D structures and the disulfide bond formed by the eight Cys-residues, we were able to predict a model for the structure of DPV-gC (Figure 4).
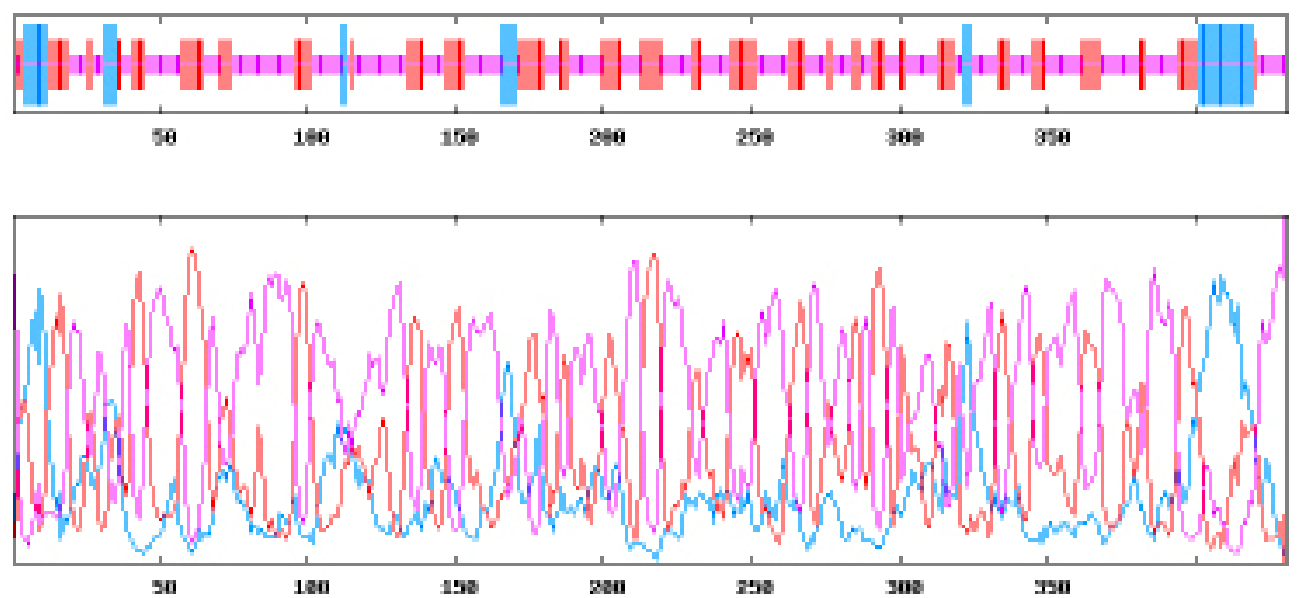

Figure 2. Secondary structure presumption of the DPV-gC. Blue represents alpha helix, red represents extended strand, and purple represents random coil.

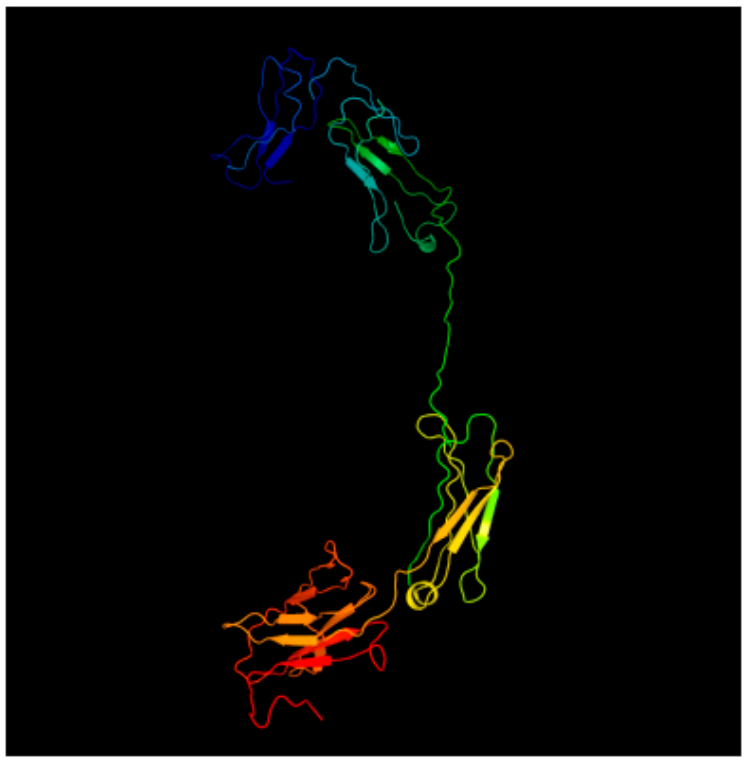

Figure 3. Prediction of DPV-gC's tertiary structure. 


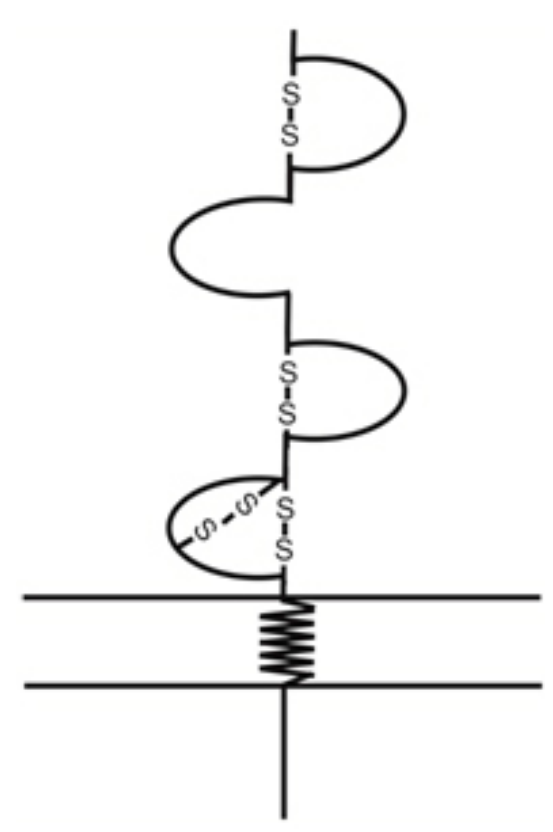

Figure 4. Model for the structure of the DPV-gC.

\section{DISCUSSION}

The aim of the present study was to provide predicted information about the structure and functions of DPV-gC, which is encoded by the newly identified DPV UL44 gene, using bioinformatic software and tools.

Results of DPV-gC sequence analysis revealed that DPV-gC has the standard membrane protein arrangement of an $\mathrm{N}$-terminal signal sequence, an external domain, a $\mathrm{C}$-terminal membrane anchor region, and a short cytoplasmic domain, and that the protein may show good immunogenicity and likely undergoes chemical modifications such as glycosylation and phosphorylation. Therefore, these results indicate that DPV-gC is a very relevant protein for the development of new antibodies and immunoassays in the clinical diagnosis of DPV.

Detailed molecular understanding plays a critical role in the improvement of vaccines. It is therefore important to determine which surface features on the DPV are responsible for inducing protective or enhancing immune responses. In this study, we focused on characterizing the B cell epitopes of DPV-gC by adopting bioinformatic approaches. The sequences at amino acids 68-71, 87-91, 369-352, and 372-374 of DPV-gC were predicted as the more prevalent dominant antigen epitopes based on multiple parameters and independent analyses in different software. The predicted dominant antigen epitopes can be used for the development of a vaccine against DPV. Further experimental studies will be performed to determine the immunogenicity and protection effect of DPV-gC peptides and to identify specific vaccine candidates. 
Comparison of the 22 alphaherpesvirus-gC protein sequences revealed that aminoterminal sequences have undergone more extensive evolutionary divergence than those at the $\mathrm{C}$-terminal half of the molecule. This appears to be a universal phenomenon for gC-like glycoproteins among all alphaherpesviruses (Allen and Coogle, 1988; Fitzpatrick et al., 1989; Nicolson and Onions, 1990). Notably, eight Cys-residue sites of the DPV-gC were found to be conserved, suggesting that these amino acids may be disulfide bond-linked and that they are likely to be important for maintaining the proper structure and functions of the protein. A structural model of HSV-1 gC demonstrated that the disulfide bond arrangement is cysteine 1 to cysteine 2, cysteine 3 to cysteine 4 , cysteine 5 to cysteine 8 , and cysteine 6 to cysteine 7 (Rux et al., 1996). The first disulfide bonds and basic and hydrophobic residues of HSV-1 gC constitute a major heparan sulfate-binding domain (Mardberg et al., 2001); the first two disulfide bonds produce loops between adjacent cysteines within the $\mathrm{C} 3 \mathrm{~b}$ binding domain (Hung et al., 1992; Rux et al., 1996), and the last two disulfide bonds result in an extended loop which may stabilize the carboxy terminus (Fitzpatrick et al., 1989; Rux et al., 1996).

The evolutionary relationship among the alphaherpesvirus-gC protein sequences was analyzed by constructing a phylogenetic tree based on the truncated DPV-gC and the truncated $\mathrm{gC}$ of 21 referenced sequences of the other alphaherpesviruses. The results revealed that DPV was clustered within a monophyletic clade, despite its close evolutionary relationship with the genus Mardivirus, which contains HVT, MDV-1, and MDV-2. These results should be useful for DPV classification studies and indicate that DPV should be placed in a separate, single genus within the Alphaherpesvirinae.

Extended strand and random coils are relatively more non-cohesive flexibility structures, which always contain B cell dominant epitopes. The alpha-helix and beta-fold of the protein have higher chemical bonding energies, which can firmly maintain proteinic higher structures, but are difficult to bind antibody, and because they are usually located at the protein interior, they seldom become B cell epitopes. In contrast, the extended strand and random coil of the protein are more loose in texture, which results in easy retortion, and can therefore stretch out of the proteinic surface and bind antibody, enabling them to become B cell epitopes (Barlow et al, 1986; Apostolopoulos et al, 2002). Therefore, secondary structure predictions of proteins are generally applied to definite B cell epitopes as an assistance medium. Combining the results of antigenic epitopes estimation and secondary structure prediction, four B cell dominant epitopes could be initially defined in DPV-gC, which were located at amino acids 68-71, 87-91, 369-352, and 372-374.

The function of a protein depends on its tertiary structure. If this is disrupted, the protein is denatured, and it loses its activity. For many proteins and peptides, disulfide bridges are a prerequisite for their proper biological function. The eight conservative Cys-residue sites in $\mathrm{DPV}-\mathrm{gC}$, which were clustered around several other conserved residues, and the general pattern of relatively low $\mathrm{N}$-terminal conservation and higher $\mathrm{C}$-terminal conservation of the $\mathrm{gC}$ like glycoproteins of all alphaherpesviruses, indicated that these glycoproteins have retained a common carboxy-terminal, disulfide bond-based framework in spite of their amino-terminal heterogeneity (Fitzpatrick et al., 1989).

In conclusion, in order to characterize the role of glycoprotein $C$ encoded by the newly identified UL44 gene of duck plague virus, bioinformatic and molecular approaches were used to predict and analyze its structure and functions. Together, these results provide necessary data for further DPV-gC research, the establishment of new types of clinical diagnoses of DPV, and for the development of a new DPV vaccine. 


\section{ACKNOWLEDGMENTS}

Research supported by grants from the Major State Basic Research Development Program of China ("973” Program) (\#2011CB111606), the National Natural Science Foundation of China (\#31072157), the Innovative Research Team Program in Education Department of Sichuan Province (\#12TD005), the Science and Technology Support Programs of Sichuan Province, China (\#2010HH0013, \#2011ZO0034, and \#2011JO0040), and the China Agricultural Research System (CARS-43-8).

\section{REFERENCES}

Allen GP and Coogle LD (1988). Characterization of an equine herpesvirus type 1 gene encoding a glycoprotein (gp13) with homology to herpes simplex virus glycoprotein C. J. Virol. 62: 2850-2858.

Apostolopoulos V, Yu M, Corper AL, Teyton L, et al. (2002). Crystal structure of a non-canonical low-affinity peptide complexed with MHC class I: a new approach for vaccine design. J. Mol. Biol. 318: 1293-1305.

Barlow DJ, Edwards MS and Thornton JM (1986). Continuous and discontinuous protein antigenic determinants. Nature 322: 747-748.

Ben-Porat T, DeMarchi JM, Lomniczi B and Kaplan AS (1986). Role of glycoproteins of pseudorabies virus in eliciting neutralizing antibodies. Virology 154: 325-334.

Blom N, Gammeltoft S and Brunak S (1999). Sequence and structure-based prediction of eukaryotic protein phosphorylation sites. J. Mol. Biol. 294: 1351-1362.

Chowdhury SI, Lee BJ, Onderci M, Weiss ML, et al. (2000). Neurovirulence of glycoprotein C(gC)-deleted bovine herpesvirus type-5 (BHV-5) and BHV-5 expressing BHV-1 gC in a rabbit seizure model. J. Neurovirol. 6: 284-295.

Combet C, Blanchet C, Geourjon C and Deleage G (2000). NPS@: network protein sequence analysis. Trends Biochem. Sci. 25: $147-150$

Davison AJ, Eberle R, Ehlers B, Hayward GS, et al. (2009). The order Herpesvirales. Arch. Virol. 154: 171-177.

Fitzpatrick DR, Babiuk LA and Zamb TJ (1989). Nucleotide sequence of bovine herpesvirus type 1 glycoprotein gIII, a structural model for gIII as a new member of the immunoglobulin superfamily, and implications for the homologous glycoproteins of other herpesviruses. Virology 173: 46-57.

Friedman HM, Wang L, Fishman NO, Lambris JD, et al. (1996). Immune evasion properties of herpes simplex virus type 1 glycoprotein gC. J. Virol. 70: 4253-4260.

Guo PX, Goebel S, Davis S, Perkus ME, et al. (1989). Expression in recombinant vaccinia virus of the equine herpesvirus 1 gene encoding glycoprotein gp13 and protection of immunized animals. J. Virol. 63: 4189-4198.

Hung SL, Srinivasan S, Friedman HM, Eisenberg RJ, et al. (1992). Structural basis of C3b binding by glycoprotein C of herpes simplex virus. J. Virol. 66: 4013-4027.

Jarosinski KW and Osterrieder N (2010). Further analysis of Marek's disease virus horizontal transmission confirms that $\mathrm{U}(\mathrm{L}) 44(\mathrm{gC})$ and $\mathrm{U}(\mathrm{L}) 13$ protein kinase activity are essential, while $\mathrm{U}(\mathrm{S}) 2$ is nonessential. J. Virol. 84: 7911-7916.

Karplus PA and Schulz GE (1985). Prediction of chain flexibility in proteins. Naturwissenschaften 72: 212-213.

Kelley LA and Sternberg MJ (2009). Protein structure prediction on the Web: a case study using the Phyre server. Nat. Protoc. 4: 363-371.

King AMQ, Lefkowitz E, Adams MJ and Carstens EB (2011). Virus Taxonomy: Ninth Report of the International Committee on Taxonomy of Viruses. Elsevier Academic Press, San Diego.

Kyte J and Doolittle RF (1982). A simple method for displaying the hydropathic character of a protein. J. Mol. Biol. 157: 105-132.

Mardberg K, Trybala E, Glorioso JC and Bergstrom T (2001). Mutational analysis of the major heparan sulfate-binding domain of herpes simplex virus type 1 glycoprotein C. J. Gen. Virol. 82: 1941-1950.

Nicolson L and Onions DE (1990). The nucleotide sequence of the equine herpesvirus $4 \mathrm{gC}$ gene homologue. Virology 179: 378-387.

Pellett PE and Roizman B (2007). The Family Herpesviridae: A Brief Introduction. In: Fields Virology. (Knipe DMandHowley PM, ed.). Lippincott Williams \& Wilkins, Philadelphia, 2480-2500.

Petersen TN, Brunak S, von Heijne G and Nielsen H (2011). SignalP 4.0: discriminating signal peptides from transmembrane regions. Nat. Methods 8: 785-786.

Rux AH, Moore WT, Lambris JD and Abrams WR (1996). Disulfide bond structure determination and biochemical 
analysis of glycoprotein C from Herpes Simplex Virus. J. Virol. 70: 5455-5465.

Sandhu TS and Shawky SA (2008). Duck Virus Enteritis (Duck Plague) In: Diseases of Poultry (Saif YM, Fadly AM and Glisson JR, eds.). Blackwell Publishing, Ames, 384-393.

Schreurs C, Mettenleiter TC, Zuckermann F, Sugg N, et al. (1988). Glycoprotein gIII of pseudorabies virus is multifunctional. J. Virol. 62: 2251-2257.

Tewari D, Nair SV, De Ungria MC, Lawrence GL, et al. (1995). Immunization with glycoprotein C of equine herpesvirus-1 is associated with accelerated virus clearance in a murine model. Arch. Virol. 140: 789-797.

Yu NY, Wagner JR, Laird MR, Melli G, et al. (2010). PSORTb 3.0: improved protein subcellular localization prediction with refined localization subcategories and predictive capabilities for all prokaryotes. Bioinformatics 26: 1608-1615. 